\title{
Improving pedestrian dynamics modelling using fuzzy logic
}

\author{
Phillip Tomé ${ }^{1}$, François Bonzon ${ }^{1}$, Bertrand Merminod ${ }^{1}$, and Kamiar \\ Aminian $^{2}$ \\ 1 EPFL - Geodetic Engineering Lab (TOPO) \\ Station 18 - 1015 Lausanne - Switzerland \\ E-mail: bertrand.merminod@epfl.ch \\ 2 EPFL - Laboratory of Movement Analysis and Measurement \\ Station 11 - 1015 Lausanne - Switzerland \\ E-mail: kamiar.aminian@epfl.ch
}

Summary. The complementary nature of MEMS based pedestrian dead-reckoning (PDR) navigation and GNSS (Global Navigation Satellite System) has long been recognised. The advantages are quite clear for those applications requiring indoor positioning and that, for one reason or another, cannot rely on short-range infrastructure-based positioning systems (e.g. WiFi, UWB) to cope with the lack of availability of GNSS indoors. One such example of application is firemen coordination during emergency interventions.

Classification of human displacement using signal pattern recognition techniques often rely on an estimation model or statistical data to compute the step length or horizontal speed information. In general, an initial calibration phase is needed which can constrain the ability to follow the quasi-erratic behaviour of a pedestrian in real time. Moreover, existing state-of-the-art PDR solutions enable only the reconstruction of the $2 \mathrm{D}$ trajectory.

This paper introduces a different approach to PDR navigation, in which pattern recognition is correlated to biomechanical principles and combined with fuzzy logic for detection and classification of a broader range of walking behaviours in 3D. Furthermore, to avoid the aforementioned limitations of stride length estimation, the step length is effectively computed by a simple inverse segment model during a specific phase of the gait cycle.

Besides a description of the algorithm, this paper includes results of a real-time implementation capable of detecting/classifying four different types of steps: forward walk, stair climbing, stair descent forward and stair descent backward. This development has been conducted in the framework of the European project LIAISON $[1,2]$ funded by the Sixth Framework Program to specifically address one of its test case scenarios, the coordination of a fire brigade intervention.

\section{Introduction}

The first applications using inertial systems were developed for the marine and aviation fields. Nowadays, every ship, aircraft and spacecraft is equipped with some form of inertial system. The inertial systems used in pedestrian 
navigation are made of Micro Electromechanical Systems (MEMS). The sensors are placed somewhere on the walker and the recorded signals are processed to derive changes in position. One of the main advantages of this approach is that the MEMS based positioning method is an independent and autonomous process that, contrary to other current position-tracking technologies, does not require any instrumented, marked or pre-mapped environment.

\subsection{Conventional vs pattern recognition PDR navigation}

Two approaches are commonly used in PDR navigation: conventional inertial navigation using zero velocity updates or classification of human displacement using signal pattern recognition techniques. Usually, the former approach consists of a classical double integration of the accelerometer signals, that allows the computation of the travelled distance. However, this distance is affected by sensors noise and drift when sampling over a long period of time. While this method is appropriate when using high-grade sensors, it performs poorly when using low-cost sensors such as MEMS [3, 4].

The second approach, being less sensitive to the quality of the sensors, has to deal with the complexities of the human movement. Body motion induces inertial acceleration and a variable gravitational component due to the change of segment inclination with respect to the vertical axis. So, by attaching a MEMS sensors box to the walker's body, usually at the hip level, it is possible to associate the recorded data with signals signatures that enable to identify and quantify body displacements.

\subsection{State of the art solutions in gait analysis}

The Laboratory of Measurement and Movement Analysis (LMAM) of EPFL has developed monitoring devices (Physilog $®$ and ASUR) and methods using MEMS sensors attached to body sites in the context of several bioengineering research programs. A patented method [5] has been introduced for monitoring physical activity based on identification of postural transition. In addition, an original and patented algorithm was proposed which computes the values of spatio-temporal gait parameters from angular velocity of the lower limbs [6] (see Fig. 1).

The state of the art solutions in PDR navigation compute the step length thanks to estimation models or statistical data [7], and do not deal with vertical displacement in $3 \mathrm{D}$. We introduce a method to effectively compute the stride length using a simple inverse pendulum model, and a classification of a broader range of walking behaviours in 3D using fuzzy logic. 


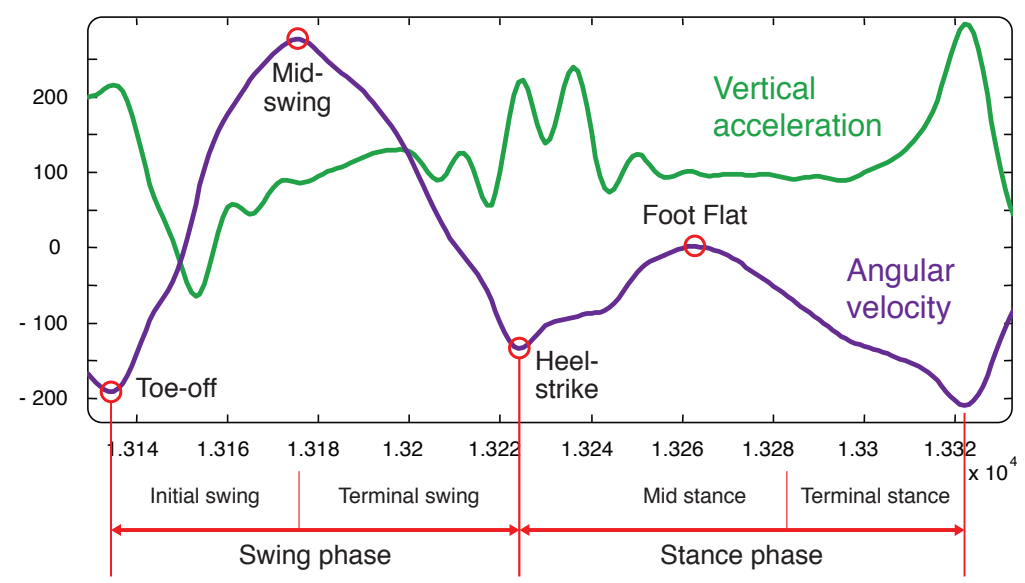

Fig. 1. Signals from a shank MEMS sensor during a full gait cycle, showing the main gait events and phases.

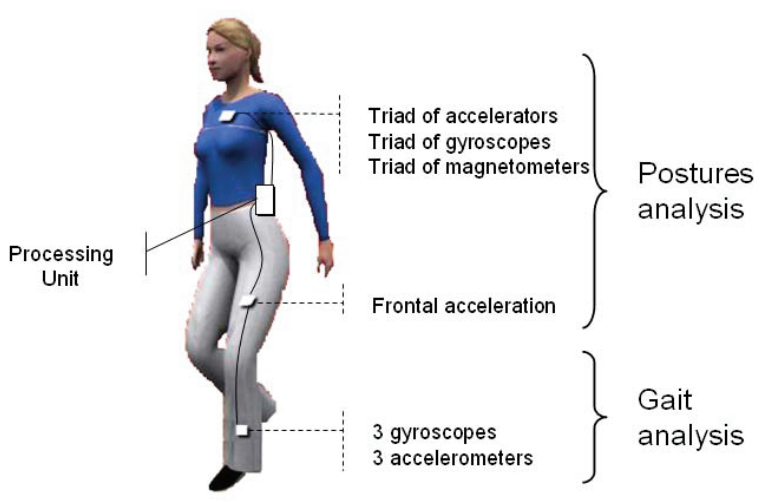

Fig. 2. Distributed MEMS sensors architecture.

\section{Gait Analysis}

In the framework of the LIAISON project, we intend to develop a novel approach to pedestrian navigation. By placing the MEMS sensors in specific body parts, one may be able to infer specific movements for navigation purposes. Based on EPFL's past research activities, three main placements were identified: the trunk, the thigh and the shank, as illustrated in Fig. 2. The top part of the body is essentially useful for postures detection and pedestrian orientation determination, while the lower part of the body offers great possibilities for gait analysis. Sensors mounted on the legs allow the computation 
of travelled displacement in the horizontal direction but also in the vertical dimension, for example when climbing stair.

\section{Step Type Classification}

The interviews of fire-fighters have allowed to focus the research effort only on basic and recurring movements. A new approach was developed on the classification of four classes of activity: flat forward walk (FW), stair ascent (SA), stair descent forward (SDF) and stair descent backward (SDB). The backward descent is a specific requirement of the fire brigade because it is safer and easier than descending in the usual forward direction. In fact, with backward descent, a fall tends to be less dangerous.

To classify the detected step into a class, an original mamdani fuzzy classification system was used (see Fig. 3). Based on the periods of the activities so far, the whole period of monitoring was divided into a list of several smaller periods and parameters. Each of these smaller periods or parameters was a fuzzy input variable and was passed to the fuzzy classifier to decide the type of activity:

- FFV The value of the shank velocity at the Foot Flat time. Determined as the maximum peak of the shank velocity between the Heel-Strike and the Toe-Off of the next step.

- DAP Difference Anterior Posterior. The difference of the velocity between the Mid-Swing and the Toe-Off.

- DPA Difference Posterior Anterior. The difference of the velocity between the Heel-Strike and the Mid-Swing.

- APV Acceleration Peak value. Determined as the minimum peak of the shank acceleration between the Toe-Off and the Mid-Swing.

- APT Time of the APV, relative to Toe-off to Mid-Swing period.

- A_amp Absolute amplitude of the acceleration signal during the entire cycle.

- LastState The activity immediately before the current activity.

- MSM Mid-Stance Mean. The mean of the velocity during a small period of the mid-stance.

- Nb_succ The number of SA, SDF or SDB performed consecutively.

\section{Stride Length Computation}

During the mid-stance and the terminal phase, the leg can be modelled as a simple inverse pendulum (see Fig. 4). The distance $d$ is obtained from the angular velocity of the shank. By considering $\alpha$ the angular rotation of the shank during this phase, it is calculated from the trigonometric relation: 


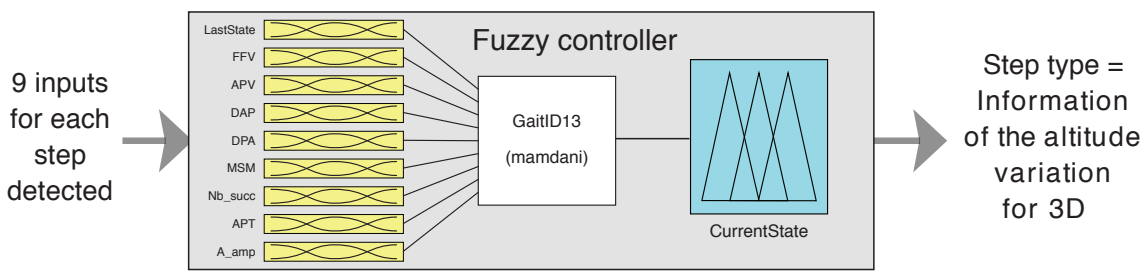

Fig. 3. The use of a fuzzy logic allows the handling of the diversity of walking patterns among different subjects, or for the same subject under different conditions.
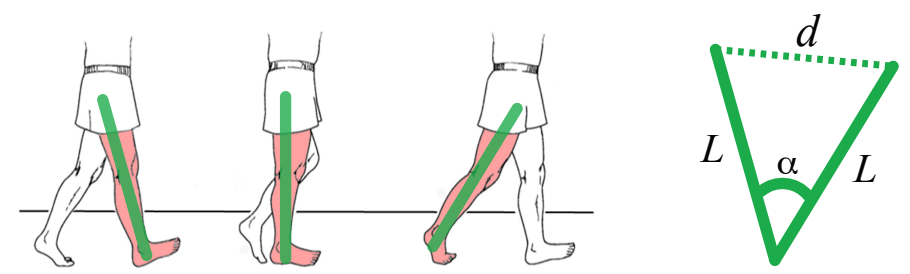

Fig. 4. Rotation of the leg during mid-stance and terminal stance.

$$
d=L \cdot \sqrt{2 \cdot[1-\cos (\alpha)]}
$$

where the angle $\alpha$ is computed by integrating the angular rate rotation of the shank during this phase, and the length of the leg $L$ is estimated by the assumption that $L=0.54$ - height of user. The mean walking velocity is then given by:

$$
V=\frac{d}{T 2-T 1}
$$

where $T 2$ is the end of the terminal stance and $T 1$ is the beginning of the mid-stance. Finally, the mean stride length corresponds to:

$$
S T=V \cdot G C T
$$

where $G C T$ is the gait cycle time.

\section{Tests and Results}

To evaluate the performance of the classification algorithms, two statistics were calculated: the sensitivity and the specificity. The sensitivity parameter is defined as the capacity of the algorithm to correctly identify the walking type, and the specificity as the ability not to provide false identification. Table 1 shows the performance of the proposed method in the detection of 


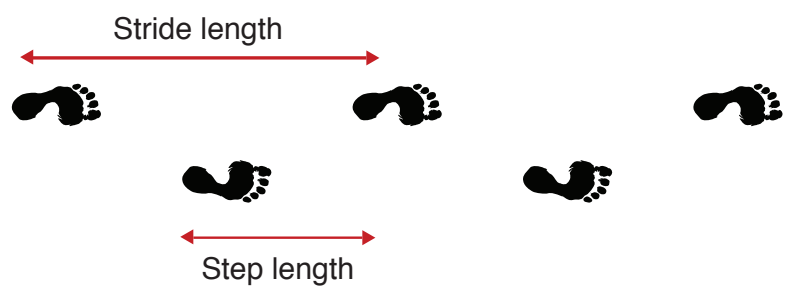

Fig. 5. The stride length is double, and not to confuse with, the step length.

basic activities during a specific experiment. The test was performed by a group of 5 healthy adults with average age of 28 years and they consisted of 5 consecutive trials for SA, SFD, SDB and FW. Except for FW, all the trials were performed on the stairs of a building composed of 66 steps and a foot flat at each 11 steps.

Table 1. Performance of the system in detecting basic walking.

\begin{tabular}{lcc}
\hline \hline & Sensitivity & Specificity \\
\hline Forward Walk (FW) & $98.1 \pm 0.6$ & $97.8 \pm 0.8$ \\
Stair Ascent (SA) & $93.5 \pm 1.7$ & $98.9 \pm 1.5$ \\
Stair Descent Forward (SDF) & $95 \pm 3.1$ & $99.1 \pm 3.2$ \\
Stair Descent Backward (SDB) & $96.1 \pm 1.2$ & $98.5 \pm 1.8$ \\
\hline
\end{tabular}

\section{Conclusion}

An original fuzzy logic classifier has been developed to improve the detection and the identification of the gait. The type of activity detected was restricted in order to focus on the basic and recurring movements of the users. Based on the results obtained, this new algorithm appears especially promising.

\section{References}

1. Valérie Renaudin, Okan Yalak, Phillip Tomé, Bertrand Merminod, Bijan Najafi, and Kamiar Aminian. D046 - EPFL contribution on MEMS based positioning. Technical report, LIAISON Consortium Deliverable, 2006.

2. Valérie Renaudin, Okan Yalak, Phillip Tomé, Bertrand Merminod, and Kamiar Aminian. D077 - EPFL contribution on MEMS-AGPS hybridisation. Technical report, LIAISON Consortium Deliverable, 2007.

3. R.G. Stirling. Development of a pedestrian navigation system using shoe mounted sensors. Master's thesis, University of Alberta, 2004. 
4. E. Foxlin. Pedestrian tracking with shoe-mounted inertial sensors. IEEE Computer Graphics and Applications, 2005.

5. B. Najafi and K. Aminian. Body movement monitoring system and method. Patent EP 00810 920.9, October 2000.

6. K. Aminian, C. Bula, P.-F. Leyvraz, and Ph. Robert. Spatio-temporal parameters of gait measured by an ambulatory system using miniature gyroscopes. Biomech, 35:689-99, 2002.

7. Q. Ladetto. Capteurs et algorithmes pour la localisation autonome en mode pédestre. PhD thesis, Ecole Polytechnique Fédérale de Lausanne, 2002. 